\title{
On the $K$-theory of the Projective Symplectic Groups
}

\author{
By \\ Haruo MinAmi* \\ Introduction
}

In this paper we compute the real $K$-groups $K O^{*}(P S p(n))$ of the projective symplectic groups $P S p(n)$.

As for the complex $K$-groups, we have in $[6,8]$ two kinds of methods of computing $K^{*}(G)$ in general for a compact connected Lie group $G$ with finite fundamental group of prime order and by using actually those methods its ring structure is explicitly described. Neither of them deals with the projective unitary groups $P U(n)$ except the case when $n$ is prime. In more general, however, the case when $n$ is a power of prime is investigated in more earlier times but not explicitly [18]. The computation in any case is based on the fact [7] that $K^{*}\left(G_{0}\right)$ (where $G_{0}$ is simply connected) is an exterior algebra generated by elements of degree one arising from the basic irreducible complex representations of $G_{0}$.

It seems not easy to find a comprehensive method of computing $K O^{*}(G)$ as in the complex case. So we proceed case by case and determined the $K O$-groups of $S O(n), P E_{6}, P E_{7}$, and $P S p\left(2^{4 n}\right)$ in $[10,11,12,13,14,15]$. Then we also use essentially the structure theorem on $K O^{*}\left(G_{0}\right)$ [17] analogous to that on $K^{*}\left(G_{0}\right)$.

We compute $K O^{*}(P S p(n))$ by applying the modification of the method used for the computation of $K O^{*}\left(P S p\left(2^{4 n}\right)\right)$. Making use of the equivariant $K O$-theory $K O_{Z / 2}^{*}$, especially the Thom isomorphism theorem for $K O_{Z / 2}$-theory, we reduce the structure of $K O^{*}(P S p(n))$ to those of $K O^{*}(S p(n))$ and $K O^{*}\left(P^{\ell}\right)$ for a certain integer $\ell>0$ where $P^{\ell}$ denotes a real projective $\ell$-space. Then we need $K^{*}(P S p(n))$ with a basis in the form conforming to our method and so we begin by computing this group by a way similar to that used in computing $K O^{*}(P S p(n))$.

In $\S \S 1-3$ we collect materials for the computations of the $K$-groups of $P S p(n)$

Communicated by K. Saito, March 28, 1995.

1991 Math. Subject Classifications: 55N15 (55R50, 57S15)

* Department of Mathematics, Nara University of Education, Takabatake-cho, Nara 630, Japan. 
which are done in $\$ \$ 4-6$. The main theorem is Theorem 5.1 .

Throughout this paper $K O^{*}(X)$ is identified with the Real $K$-group $K R^{*}(X)$ in the sense of Atiyah [1] because the reality of all spaces is assumed to be trivial.

\section{§ 1. Preliminaries}

From now on, we denote by $G=S p(n)$ the symplectic group on quaternionic $n$-space $\boldsymbol{H}^{n}$ and by $\Gamma$ the center of $G$ which is a cyclic subgroup of order 2 generated by $-I$ where $I$ is the identity of $G$. Then $P G=G / \Gamma$.

By $H$ we denote the representation space of the canonical nontrivial 1dimensional real representation of $\Gamma$ and by $p H$ the direct sum of $p$ copies of $H$. Let $B\left(p H \oplus \boldsymbol{R}^{q}\right)$ and $S\left(p H \oplus \boldsymbol{R}^{q}\right)$ be the unit ball and the unit sphere in $p H \oplus \boldsymbol{R}^{q}$ centered at the origin $o$, and let $\Sigma^{p H-q}=B\left(p H \oplus \boldsymbol{R}^{q}\right) / S\left(p H \oplus \boldsymbol{R}^{q}\right)$ be the quotient space with the collapsed $S\left(p H \oplus \boldsymbol{R}^{q}\right)$ as a base point. These spaces are equipped with the induced $\Gamma$-action and $P^{k}=S((k+1) H) / \Gamma$, the real projective $k$-space.

Let $L=H \otimes C$ be the complexification of $H$. We write $L$ for $2 H$ in the complex case since $L \approx 2 H$ as a $\Gamma$-space.

Let $p H \subset(p+q) H$ be the submodule consisting of the first $p$-tuples. Then the assignment $(\boldsymbol{x}, \boldsymbol{y}) \mapsto(\boldsymbol{x}, \mathbf{y} /|\boldsymbol{y}|)(\boldsymbol{x} \in p H, \boldsymbol{y} \in q H)$ induces an equivariant homeomorphism $S((p+q) H)-S(p H) \approx \operatorname{Int} B(p H) \times S(q H)$. Taking one-point compactification of the spaces on both sides yields an equivariant homeomorphism

$$
S((p+q) H) / S(p H) \approx \Sigma^{p H} \wedge S(q H)_{+}
$$

where $X_{+}$denotes the disjoint union of $X$ and a single point + which is to serve as the base point for $X_{+}$.

To compute $K O^{*}(P G)$ we use the $K$-functor $K S p$ in addition to $K$. Here $K S p(X)$ denotes the $K$-group associated with the quaternionic vector bundles over $X$, and a quaternionic vector bundle means a complex vector bundle provided with a complex conjugate anti-involution. Given two quaternionic vector bundles $E$ $\rightarrow X$ and $F \rightarrow Y$, the external tensor product $E \hat{\otimes}_{C} F \rightarrow X \times Y$ becomes a Real vector bundle in the sense of [1], so that this can be viewed as a usual real vector bundle. Thus any given $x \in \widetilde{K S} p(X)$ and $y \in \widetilde{K S} p(Y)$ give rise to an element of $\widetilde{K O}(X \wedge Y)$, denoted by $x \wedge_{c} y$, for based spaces $X$ and $Y$.

Let $f: X \rightarrow S p(n)$ be a base point preserving map from a based space $X$ to $S p(n)$ with $I$ as base point. Then the homotopy class of $f$ defines in a canonical manner an element of $\widetilde{K S} p^{-1}(X)$ which we denote by $\beta(f)$. Of course a similar fact holds for $K O$ - and $K$-theories. We use the same symbol $\beta($ ) for such elements.

Identify $S^{0}=O(1), S^{1}=U(1)$ and $S^{3}=S p(1)$, and let $\iota_{k}$ denote the identity map of $S^{k}$ into itself for $k=0,1,3$, then each $\beta\left(\iota_{k}\right)$ is a generator of the $(-1)$ dimensional coefficient group of the corresponding $K$-theory. We write $\eta_{1}=\beta\left(\iota_{0}\right), \mu=\beta\left(\iota_{1}\right)$ and $\sigma=\beta\left(\iota_{3}\right)$. And moreover we put 


$$
\eta_{8}=\sigma \wedge_{C} \sigma, \eta_{4}=\epsilon_{H} \wedge_{C} \sigma
$$

where $\epsilon_{H}$ denotes the reduced bundle of the trivial quaternionic line bundle over $S^{0}$. Then $K^{*}(+)=\boldsymbol{Z}[\mu] /\left(\mu^{2}-1\right), K O^{*}(+)=\boldsymbol{Z}\left[\eta_{1}, \eta_{4}, \eta_{8}\right] /\left(2 \eta_{1}, \eta_{1}^{2}, \eta_{1} \eta_{4}, \eta_{4}^{2}-4\right.$, $\left.\eta_{8}-1\right)$ and $K S p^{*}(X) \cong K O^{*}(X)$ which is induced by smash multiplication $\wedge_{c}$ by $\sigma$. Further there holds $s(\sigma)=\mu^{2}$ where $s: K \operatorname{Sp}^{*}(X) \rightarrow K^{*}(X)$ denotes the complexification homomorphism.

Usually the functor $K$ is $\boldsymbol{Z} / 2$-graded as shown above, but it is considered to be $\boldsymbol{Z} / 8$-graded, that is, the order of $\mu$ is 4 , in the case of connecting two functors $K$ and $K O$. In fact we use the following exact sequence of Bott [2] :

$$
\cdots \rightarrow K O^{*}(X) \stackrel{x}{\rightarrow} K O^{*}(X) \stackrel{c}{\rightarrow} K^{*}(X) \stackrel{\delta}{\rightarrow} K O^{*}(X) \rightarrow \cdots
$$

where $\chi$ is the multiplication by $\eta_{1}, c$ the complexification homomorphism and $\delta$ the homomorphism given by the equality $\delta(x)=r\left(\mu^{-1} x\right)$ using the realification homomorphism $r: K^{*}(X) \rightarrow K O^{*}(X)$.

We now confine ourself to the real and complex $K$-theories. It is well known that there holds $K O_{\Gamma}^{*}(X) \cong K O^{*}(X / \Gamma)$ if $X$ is a free $\Gamma$-space. Let $R O(\Gamma)$ and $R(\Gamma)$ be the real and complex representation rings of $\Gamma$. Then we also have $K O_{\Gamma}^{*}$ $(X) \cong R O(\Gamma) \otimes K O^{*}(X)$ if $X$ is a trivial $\Gamma$-space by reason of $\Gamma \cong Z / 2$. Similar isomorphisms hold for the complex $K$-functor (of course $R O(\Gamma)$ must be replaced by $R(\Gamma)$ ). In the sequel we assume that such isomorphisms are identified.

Recall from $[16,3,9]$ the Thom isomorphism theorem which is our basic tool together with the facts stated in the next section.

$$
\text { For } k \geq 0, \epsilon=0,1 \text { there exists elements }
$$

$$
\tau_{k L} \in \tilde{K}_{\Gamma}\left(\Sigma^{k L}\right) \text { and } \omega_{(8 k+4 \epsilon) H+4 \epsilon} \in \widetilde{K O_{\Gamma}}\left(\Sigma^{(8 k+4 \epsilon) H+4 \epsilon}\right)
$$

satisfying the following properties:

(i) Multiplication by $\tau_{k L}$ and $\omega_{(8 k+4 \epsilon) H+4 \epsilon}$ induces isomorphisms $K_{\Gamma}^{*}(X) \cong$ $\tilde{K}_{\Gamma}\left(\Sigma^{k L} \wedge X_{+}\right)$and $K O_{\Gamma}^{*}(X) \cong \widetilde{K O_{\Gamma}^{*}}\left(\Sigma^{(8 k+4 \epsilon) H+4 \epsilon} \wedge X_{+}\right)$for any $\Gamma$-space $X$ respectively.

(ii) $j^{*}\left(\tau_{k L}\right)=2^{k-1}(1-L) \in K_{\Gamma}(o)=R(\Gamma), j^{*}\left(\omega_{(8 k+4 \epsilon) H+4 \epsilon}\right)=2^{4 k-1}(1-H)^{1+\epsilon} \eta_{4}^{\epsilon}$ $\in \widetilde{K O_{\Gamma}}\left(\Sigma^{4 \epsilon}\right)=R O(\Gamma) \cdot \eta_{4}^{\epsilon}, \psi\left(\tau_{k L}\right)=\mu^{k}$ and $\phi\left(\omega_{(8 k+4 \epsilon) H+4 \epsilon}\right)=\eta_{8}^{k+\epsilon}$, so that $c\left(\omega_{(8 k+4 \epsilon) H+4 \epsilon}\right)=\tau_{(4 k+2 \epsilon) L} \mu^{2 \epsilon}$ where $j$ denotes the composite $\{0\} \subset B(k L) \rightarrow \Sigma^{k L}$ of obvious two maps and the inclusion $\Sigma^{4 \epsilon} \subset \Sigma^{(8 k+4 \epsilon) H+4 \epsilon}$ and $\phi$ denotes the forgetful transformations $K_{\Gamma} \rightarrow K$ and $\mathrm{KO}_{\Gamma} \rightarrow \mathrm{KO}$.

\section{§ 2. The $K$-groups of $G$ and $P^{k}$}

In this section we recall the ring structure of the $K$-groups of $G$ and give that 
of the $K$-groups of $P^{k}$ which we need later on.

Let $\bar{\rho}=i d: G \rightarrow S p(n)$ be the canonical quaternionic representation of $G$ and let $\rho: G \rightarrow U(2 n)$ denote the complexification of $\bar{\rho}$. Then we know (2.1) and (2.2) below from [7] and [17] respectively.

$$
K^{*}(G)=\Lambda\left(\beta(\rho), \beta\left(\lambda^{2} \rho\right), \cdots, \beta\left(\lambda^{n} \rho\right)\right)
$$

as a ring where $\lambda^{k} \rho$ denotes the $k$-th exterior power of $\rho$.

Let $\lambda_{C}^{k} \bar{\rho}$ be the $k$-th exterior power of $\bar{\rho}$ over $C$. As seen in the previous section $\lambda_{C}^{2 l} \bar{\rho}$ is real and $\lambda_{C}^{2 f+1} \bar{\rho}$ is quaternionic, so that we have $\beta\left(\lambda_{C}^{2 l} \bar{\rho}\right) \in \widetilde{K O}^{-1}(G)$ and $\beta\left(\lambda_{C}^{2 j+1} \bar{\rho}\right) \in \widetilde{K S} p^{-1}(G)$. We also set $\bar{\beta}\left(\lambda_{C}^{2 j+1} \bar{\rho}\right)=\sigma \wedge_{C} \beta\left(\lambda_{C}^{2 j+1} \bar{\rho}\right) \in \widetilde{K O^{-5}}(G)$. Then we have

(2.2) $K O^{*}(G)=\Lambda_{K O^{*}(+)}\left(\beta\left(\lambda_{C}^{2 i} \bar{\rho}\right), \bar{\beta}\left(\lambda_{C}^{2 j+1} \bar{\rho}\right)(1 \leq i \leq[n / 2], 0 \leq j \leq[(n-1) / 2])\right)$

as a $K O^{*}(+)$-module where [] denotes the Gaussian notation. Further, the ring structure is given by

$$
\beta\left(\lambda_{C}^{2 \imath} \bar{\rho}\right)^{2}=\eta_{1}\left(\beta\left(\lambda_{C}^{4 l} \bar{\rho}\right)+\left(\begin{array}{c}
2 n \\
2 i
\end{array}\right) \beta\left(\lambda_{C}^{2 \imath} \bar{\rho}\right)\right) \text { and } \bar{\beta}\left(\lambda_{C}^{2 j \perp 1} \bar{\rho}\right)^{2}=\eta_{1} \beta\left(\lambda_{C}^{4 j+2} \bar{\rho}\right)
$$

where the indices $4 i$ and $4 j+2$ indicate the integers mod $2 n$ because of $\lambda_{C}^{k} \bar{\rho}$ $=\lambda_{C}^{2 n-k} \bar{\rho}$ for $0 \leq k \leq n$. (The indices appeared later on indicate such integers as well.) These square formulas follow from

$$
x^{2}=\eta_{1} \lambda^{2} x \text { for } x \in \widetilde{K O^{-1}}(X) \text { and }\left(\sigma \wedge_{C} x\right)^{2}=\eta_{1} \lambda_{C}^{2} x \text { for } x \in \widetilde{K S} p^{-1}(X)
$$

which is due to $[4,11]$.

Let $\bar{\gamma} \in \widetilde{K O}^{-1}\left(P^{k-1}\right)$ be the reduced vector bundle of the real line bundle $\xi$ : $S(k H) \times{ }_{\Gamma} H \rightarrow P^{k-1}$. That is, $\bar{\gamma}=[\xi]-1$ where [ ] denotes the equivalence class. And write $\gamma=c(\bar{\gamma}) \in \tilde{K}\left(P^{k-1}\right)$. Let

$$
\nu=p^{*}\left(\mu^{k}\right) \in \tilde{K}^{-1}\left(P^{2 k-1}\right) \text { and } \bar{\nu}=p^{*}\left(\eta_{8}^{k}\right) \in \widetilde{K O}^{-\ell}\left(P^{8 k-\ell}\right)
$$

where $p$ denotes the map $P^{k} \rightarrow S^{k}$ obtained by collapsing the outside of a top dimensional cell in $P^{k}$. From [2] we then have

(2.4) $\tilde{K}\left(P^{2 r-1}\right)=\boldsymbol{Z} / 2^{r-1} \cdot \gamma, \tilde{K}^{-1}\left(P^{2 r-1}\right)=\boldsymbol{Z} \cdot \nu, \tilde{K}\left(P^{2 r}\right)=\boldsymbol{Z} / 2^{r} \cdot \gamma, \tilde{K}^{-1}\left(P^{2 r}\right)=0$

where the relations $\gamma^{2}+2 \gamma=0$ and $\nu^{2}=0$ hold.

As for $K O^{*}\left(P^{k-1}\right)$ it has been additively determined in $[5,19]$ for any $k \geq 2$. By using (1.2) together with this result and (2.4), and by observing the exact sequence for the pair $(B(k L), S(k L))$ in $K O_{\Gamma^{-}}$-theory together with (1.4), we 
can get the multiplicative structure of them relevant to our calculation as follows.

(2.5) In the following table the modules $\widetilde{K O^{-p}}\left(P^{8 r+q}\right)$ are arranged in the order subordinate to $p=0,1, \cdots, 7$ for each $q$.

Case $q=3$.

$$
\begin{aligned}
& \boldsymbol{Z} / 2^{4 r+2} \cdot \bar{\gamma}, \boldsymbol{Z} \cdot \eta_{4} \bar{\nu} \oplus \boldsymbol{Z} / 2 \cdot \eta_{1} \bar{\gamma}, \boldsymbol{Z} / 2 \cdot \eta_{1}^{2} \bar{\gamma}, 0, \boldsymbol{Z} / 2^{4 r} \cdot \eta_{4} \bar{\gamma} \\
& \boldsymbol{Z} \cdot \bar{\nu}, \boldsymbol{Z} / 2 \cdot \eta_{1} \bar{\nu} \oplus \boldsymbol{Z} / 2 \cdot \zeta, \boldsymbol{Z} / 2 \cdot \eta_{1}^{2} \bar{\nu} \oplus \boldsymbol{Z} / 2 \cdot \eta_{1} \boldsymbol{\zeta}
\end{aligned}
$$

where $\zeta$ is given by $c(\zeta)=2^{4 r} \mu^{3} \gamma$ and the generators are subject to the relations:

$$
\bar{\gamma}^{2}+2 \bar{\gamma}=0, \bar{\nu}^{2}=\zeta^{2}=\eta_{4} \zeta=0, \eta_{1} \bar{\nu}=\bar{\gamma} \zeta, \eta_{1}^{2} \zeta=2^{4 r+1} \bar{\gamma}
$$

Case $q=4$.

$$
\boldsymbol{Z} / 2^{4 r+3} \cdot \bar{\gamma}, \boldsymbol{Z} / 2 \cdot \eta_{1} \bar{\gamma}, \boldsymbol{Z} / 2 \cdot \eta_{1}^{2} \bar{\gamma}, 0, \boldsymbol{Z} / 2^{4 r+1} \cdot \eta_{4} \bar{\gamma}, 0, \boldsymbol{Z} / 2 \cdot \boldsymbol{\zeta}, \boldsymbol{Z} / 2 \cdot \eta_{1} \zeta
$$

where $\zeta$ is given by $c(\zeta)=2^{4 r+1} \mu^{3} \gamma$ and the generators are subject to the relations:

$$
\bar{\gamma}^{2}+2 \bar{\gamma}=0, \zeta^{2}=\eta_{4} \zeta=0, \bar{\gamma} \zeta=0, \eta_{1}^{2} \zeta=2^{4 r+2} \bar{\gamma}
$$

Case $q=5$.

$$
\boldsymbol{Z} / 2^{4 r+3} \cdot \bar{\gamma}, \boldsymbol{Z} / 2 \cdot \eta_{1} \bar{\gamma}, \boldsymbol{Z} / 2 \cdot \eta_{1}^{2} \bar{\gamma}, \boldsymbol{Z} \cdot \bar{\nu}, \boldsymbol{Z} / 2^{4 r+2} \cdot \eta_{4} \bar{\gamma}, 0(p=5,6), \boldsymbol{Z} \cdot \overline{\boldsymbol{\nu}}^{\prime}
$$

where $\bar{\nu}^{\prime}$ is given by $c\left(\bar{\nu}^{\prime}\right)=\mu^{3} \nu$ and the generators are subject to the relations:

$$
\bar{\gamma}^{2}+2 \bar{\gamma}=0, \quad \bar{\nu} \bar{\nu}^{\prime}=0, \eta_{1} \bar{\nu}=2^{4 r+1} \eta_{4} \bar{\gamma}, \eta_{1} \bar{\nu}^{\prime}=2^{4 r+2} \bar{\gamma}, \eta_{4} \bar{\nu}=2 \bar{\nu}^{\prime}, \eta_{4} \bar{\nu}^{\prime}=2 \bar{\nu} .
$$

Case $q=6$.

$\boldsymbol{Z} / 2^{4 r+3} \cdot \bar{\gamma}, \boldsymbol{Z} / 2 \cdot \eta_{1} \bar{\gamma}, \boldsymbol{Z} / 2 \cdot \eta_{1}^{2} \bar{\gamma} \oplus \boldsymbol{Z} / 2 \cdot \zeta, \boldsymbol{Z} / 2 \cdot \eta_{1} \zeta, \boldsymbol{Z} / 2^{4 r+3} \cdot \eta_{4} \bar{\gamma}, 0(p=5,6,7)$

where $\zeta$ is given by $c(\zeta)=2^{4 r+2} \mu \gamma$ and the generators are subject to the relations:

$$
\bar{\gamma}^{2}+2 \bar{\gamma}=0, \zeta^{2}=\bar{\gamma} \zeta=0, \eta_{1}^{2} \zeta=2^{4 r+2} \eta_{4} \bar{\gamma}
$$

Case $q=7$.

$$
\boldsymbol{Z} / 2^{4 r+5} \cdot \bar{\gamma}, \boldsymbol{Z} / 2 \cdot \eta_{1} \bar{\gamma}, \boldsymbol{Z} / 2 \cdot \eta_{1}^{2} \bar{\gamma}, \boldsymbol{Z} \cdot \overline{\boldsymbol{\nu}}^{\prime}, \boldsymbol{Z} / 2^{4 r+4} \cdot \eta_{4} \bar{\gamma}, 0(p=5,6), \boldsymbol{Z} \cdot \overline{\boldsymbol{\nu}}
$$


where $\bar{\nu}^{\prime}$ is given by $c\left(\bar{\nu}^{\prime}\right)=\mu \nu$ and the generators are subject to the relations:

$$
\bar{\gamma}^{2}+2 \bar{\gamma}=0, \bar{\nu} \bar{\nu}^{\prime}=0, \eta_{4} \bar{\nu}=2 \bar{\nu}^{\prime}, \eta_{4} \bar{\nu}^{\prime}=2 \bar{\nu}, \eta_{1} \bar{\nu}=2^{4 r+4} \bar{\gamma}, \eta_{1} \bar{\nu}^{\prime}=2^{4 r+3} \eta_{4} \bar{\gamma}
$$

\section{§ 3. Generators of $K^{*}(P G)$ and $K O^{*}(P G)$}

In this section we describe the multiplicative generators of $K^{*}(P G)$ and $K O^{*}(P G)$ except one of them in the real case which is given in $\$ \S 5$ and 6 .

For the reduced complex and real line bundles over $P G$ associated with the principal $\Gamma$-bundle $G \rightarrow P G$ we use the same symbols

$$
\gamma \in \tilde{K}(P G) \text { and } \bar{\gamma} \in \widetilde{K O}(P G)
$$

as those over $P^{k}$

Since $\lambda^{2 \imath} \rho(-I)=\lambda_{c}^{2 \imath} \bar{\rho}(-I)=I$, the $\lambda^{2 \imath} \rho$ and $\lambda_{c}^{2 \imath} \bar{\rho}$ factor through the canonical projection $\pi: G \rightarrow P G$ and so these can be viewed as representations of $P G$. Further, since $\bar{\rho}$ is quaternionic, the same argument as that on the product $\wedge_{c}$ in $\S 1$ shows that the latter is real. Thus we have

$$
\beta\left(\lambda^{2 \imath} \rho\right) \in \tilde{K}^{-1}(P G) \text { and } \beta\left(\lambda_{c}^{2 i} \bar{\rho}\right) \in \widetilde{K O}^{-1}(P G)
$$

for $1 \leq i \leq[n / 2]$.

In order to give another kind of odd dimensional generator we consider the binomial coefficients $\left(\begin{array}{c}2 n \\ 2 j+1\end{array}\right)$ 's. From now on, we denote by

$$
s=\nu_{2}(n)
$$

the exponent of 2 in the $n$. Since $2^{s+1} \mid\left(\begin{array}{c}2 n \\ 2 j+1\end{array}\right)$ for $0 \leq j \leq n-1$, we put

$$
d_{j}=\left(\begin{array}{c}
2 n \\
2 j+1
\end{array}\right) / 2^{s+1}
$$

Then evidently $d_{0}$ is odd and $d_{0}+d_{1}+\cdots+d_{n-1}=2^{2 n-s-2}$ so that $\left(d_{0}, d_{1}\right.$, $\left.\cdots, d_{n-1}\right)=1$. Hence there are integers $a_{0}, a_{1}, \cdots, a_{n-1}$ such that

$$
a_{0} d_{0}+a_{1} d_{1}+\cdots+a_{n-1} d_{n-1}=1
$$

From this fact we see that there exists a base point preserving map

$$
\bar{\kappa}: G \rightarrow \operatorname{Sp}\left(2^{s}+m\right)
$$


such that $\bar{\kappa}(-g)=D\left(-I_{1}, I_{2}\right) \bar{\kappa}(g)(g \in G)$ for some $m \geq 0$ where $I_{1} \in S p\left(2^{s}\right)$ and $I_{2} \in S p(m)$ denote the identities. Here in general we write $D\left(A_{1}, \cdots, A_{\ell}\right)$ for the matrix with square matrices $A_{1}, \cdots, A_{\ell}$ placed diagonally in this order and with zero entries in the other spaces. And when $A_{i}=A(1 \leq i \leq \ell), D\left(A_{1}, \cdots, A_{\ell}\right)$ is abbreviated as $D(\ell A)$. Then the desired map $\bar{\kappa}$ can be given as follows. We may assume without loss of generality that $a_{0}, \cdots, a_{\ell} \geq 0$ and the others are negative. Let $A_{j}(g)=D\left(a_{j} \lambda_{c}^{2 j+1} \bar{\rho}(g)\right)$ or $D\left(-a_{j} \lambda_{c}^{2 j+1} \bar{\rho}(g)\right)$ according as $a_{j} \geq 0$ or $a_{j}<0$. Then it suffices to put

$$
\bar{\kappa}(g)=D\left(I_{1}, A_{\ell+1}(g)^{-1}, \cdots, A_{n-1}(g)^{-1}\right) D\left(A_{0}(g), \cdots, A_{\ell}(g)\right) \quad(g \in G)
$$

where $I_{1}$ is as above. Suppose now that $m=0$ for simplicity.

Define a map $\bar{\kappa}_{j}: G \rightarrow S p\left(2^{s} d_{j}\right)$ for $0 \leq j \leq n-1$ by $\bar{\kappa}_{j}(g)=D\left(d, \bar{\kappa}(g)^{-1}\right)$ $\lambda_{C}^{2 j+1} \bar{\rho}(g)(g \in G)$. Then, since $\bar{\kappa}_{j}(-g)=\bar{\kappa}_{j}(g)$, the $\bar{\kappa}_{j}$ factors through $\pi: G \rightarrow P G$ and so it can be viewed as a map

$$
\bar{\kappa}_{\jmath}: P G \rightarrow \operatorname{Sp}\left(2^{s} d_{\jmath}\right)
$$

(Here note that $\bar{\kappa}_{\jmath}=\bar{\kappa}_{n-\jmath-1}$ because of $\lambda_{C}^{2 j+1} \bar{\rho}=\lambda_{C}^{2 n-2 \jmath-1} \bar{\rho}$.) Clearly these maps define $\beta(\bar{\kappa}) \in \widetilde{K S} p^{-1}(G)$ and $\beta\left(\bar{\kappa}_{\jmath}\right) \in \widetilde{K S} p^{-1}(P G)$. Let $\kappa$ and $\kappa$, denote the complexification of $\bar{\kappa}$ and $\bar{\kappa}_{3}$ respectively. Then

$$
\beta(\kappa)=c(\beta(\bar{\kappa})) \in \tilde{K}^{-1}(G) \text { and } \beta\left(\kappa_{\jmath}\right)=c\left(\beta\left(\bar{\kappa}_{\jmath}\right)\right) \in \tilde{K}^{-1}(P G)
$$

Further we set

$$
\bar{\beta}(\bar{\kappa})=\sigma \wedge_{C} \beta(\bar{\kappa}) \in \widetilde{K O}^{-5}(G) \text { and } \bar{\beta}\left(\bar{\kappa}_{\jmath}\right)=\sigma \wedge_{C} \beta\left(\bar{\kappa}_{\jmath}\right) \in \widetilde{K O}^{-5}(P G)
$$

The map $P G \rightarrow S p\left(2^{s}\right)$ given by the assignment $\pi(g) \mapsto \bar{\kappa}(g)^{2}(g \in G)$ defines similarly an element of $\widetilde{K S p^{-1}}(P G)$, denoted by $\beta(\bar{\kappa}, 2)$. And we set

$$
\beta(\kappa, 2)=c(\beta(\bar{\kappa}, 2)) \in \tilde{K}^{-1}(P G) \text { and } \bar{\beta}(\bar{\kappa}, 2)=\sigma \wedge_{c} \beta(\bar{\kappa}, 2) \in \widetilde{K O}^{-5}(P G) \text {. }
$$

By definition we can easily check that there hold the equalities

$$
\pi^{*}\left(\bar{\beta}\left(\bar{\kappa}_{j}\right)\right)=\bar{\beta}\left(\lambda_{C}^{2 \jmath+1} \bar{\rho}\right)-d_{j} \bar{\beta}(\bar{\kappa})
$$

and

$$
\bar{\beta}(\bar{\kappa})=a_{0} \bar{\beta}\left(\lambda_{C}^{1} \bar{\rho}\right)+\cdots+a_{n-1} \bar{\beta}\left(\lambda_{C}^{2 n-1} \bar{\rho}\right)
$$

so that $a_{0} \pi^{*}\left(\bar{\beta}\left(\bar{\kappa}_{0}\right)\right)+\cdots+a_{n-1} \pi^{*}\left(\bar{\beta}\left(\bar{\kappa}_{n-1}\right)\right)=0$. Let $V$ be the free submodule of 
$K O^{-5}(G)$ on $\bar{\beta}\left(\lambda_{C}^{2 j+1} \bar{\rho}\right) \quad(0 \leq j \leq[(n-1) / 2])$. Then this fact shows that the submodule $W \subset V$ generated by $\pi^{*}\left(\bar{\beta}\left(\bar{\kappa}_{j}\right)\right)$ 's has the rank smaller than $[(n-1) / 2]$ and that there exist certain integral linear combinations $\bar{\chi}_{1}, \cdots, \bar{\chi}_{t}$ of $\lambda_{C}^{2 j-1} \bar{\rho}$ 's over $\boldsymbol{Z}$ such that their characters on $\Gamma$ are zero and they yield a free basis for $W$ as follows. By the construction similar to that of $\kappa_{j}^{\prime}$ s, each $\bar{\chi}_{k}$, being viewed as a virtual representation of $P G$, gives rise to an element $\beta\left(\bar{\chi}_{k}\right) \in \widetilde{K S}_{P}^{-1}(P G)$ so that we have

$$
\beta\left(\chi_{k}\right) \in \tilde{K}^{-1}(P G) \text { and } \bar{\beta}\left(\bar{\chi}_{k}\right) \in \widetilde{K O}^{-5}(P G)
$$

for $1 \leq k \leq t$ as before. Then $\pi^{*}\left(\bar{\beta}\left(\bar{\chi}_{k}\right)\right) \quad(1 \leq k \leq t)$ form a basis for $W$ and we will find that $t$ is just equal to $[(n-1) / 2]-1$ in the sequel.

Finally for later use we give an estimate of the orders of $\bar{\gamma}$ and $\gamma$ which follows from the property of $\bar{\kappa}$ immediately.

$$
2^{s} \bar{\gamma} \otimes \boldsymbol{H}=0 \in \widetilde{K S} p(P G) \text { so that } 2^{s} \eta_{4} \bar{\gamma}=2^{s+2} \bar{\gamma}=0 \text { and } 2^{s+1} \gamma=0
$$

\section{§4. Calculation of $K^{*}(P G)$}

In this section we prove the following

Theorem 4.1 ( $[6,8])$. With the above notation

$$
K^{*}(P S p(n))=\boldsymbol{Z}[\gamma] /\left(\gamma^{2}+2 \gamma, 2^{s+1} \gamma\right)
$$

$$
\otimes \Lambda\left(\beta(\kappa, 2), \beta\left(\lambda^{2 i} \rho\right), \beta\left(\chi_{j}\right)(1 \leq i \leq[n / 2], 1 \leq j \leq[(n-1) / 2]-1)\right) / I
$$

where $I=(\gamma \otimes \beta(\kappa, 2))$.

Apply the $K$-functor $K_{\Gamma}$ to the cofibration $S((s+2) L) \times G \stackrel{i}{\rightarrow} B((s+2) L)$ $\times G \stackrel{j}{\rightarrow} \Sigma^{(s+2) L} \wedge G_{+}$where $i$ and $j$ are the obvious inclusion and projection and $s$ is the integer as in previous section. Then we have an exact sequence

$$
\cdots \rightarrow \tilde{K}_{\Gamma}^{*}\left(\Sigma^{(s+2) L} \wedge G_{\perp}\right) \stackrel{j^{*}}{\rightarrow} K_{\Gamma}^{*}(B((s+2) L) \times G) \stackrel{i^{*}}{\rightarrow} K^{*}(S((s+2) L) \times G) \stackrel{\delta}{\rightarrow} \cdots
$$

where $\delta$ denotes the coboundary homomorphism. By (1.4)(ii) and (3.1) we get $j^{*}\left(\tau_{(s+2) L} \wedge 1\right)=-2^{s+1} \gamma=0 \in K_{\Gamma}^{*}(B((s+2) L) \times G)=K^{*}(P G)$. Hence, via the Thom isomorphism $\tilde{K}_{\Gamma}^{*}\left(\Sigma^{(s+2) L} \wedge G_{+}\right) \cong K^{*}(P G)$, this sequence becomes

$$
0 \rightarrow K^{*}(P G) \stackrel{I}{\rightarrow} K_{\Gamma}^{*}(S((s+2) L) \times G) \stackrel{\Delta}{\rightarrow} K^{*}(P G) \rightarrow 0
$$

where the equality 


$$
\Delta(x I(y))=\Delta(x) y
$$

holds because the equality $\delta\left(x i^{*}(y)\right)=\delta(x) y$ does.

Next we consider the group $K_{\Gamma}^{*}(S((s+2) L) \times G)$ of (4.2) and begin by preparing generators of this group. Let $\tilde{\gamma}=p_{1}^{*}(\gamma), \tilde{\nu}=p_{1}^{*}(\nu)$ and $\tilde{\beta}\left(\lambda^{2 i} \rho\right)=p_{2}^{*}$ $\left(\beta\left(\lambda^{2 i} \rho\right)\right)$ for $0 \leq i \leq n$ where $p_{1}$ and $p_{2}$ are the canonical projections from $S((s+2) L) \times G$ to the 1 st and 2nd factors.

By (2.4) $2^{s+1} \gamma=0 \in \tilde{K}\left(P^{2 s+3}\right)$. This fact shows that there is a $\Gamma$-bundle isomorphism $S((s+2) L) \times\left(2^{s+1} L \oplus M\right) \cong S((s+2) L) \times\left(C^{2^{s+1}} \oplus M\right)$ for some $\Gamma$ -module $M$. Here we may assume that this isomorphism is chosen such that the Thom class $\tau_{(s+2) L}$ arises from this in the usual way. Now this gives rise to a base point preserving map $\alpha: S((s+2) L) \rightarrow U\left(2^{s+1}+m\right)$ for some $m \geq 0$ satisfying $\alpha(-x)=\alpha(x) D\left(-I_{1}, I_{2}\right)(x \in S((s+2) L))$ where $I_{1} \in U\left(2^{s+1}\right)$ and $I_{2} \in U(m)$ denote the identities. Assuming that $m=0$ for simplicity we can define a map $f_{j}: S((s+2) L) \times G \rightarrow U\left(2^{s+1} d_{j}\right)$ for $0 \leq j \leq n-1$ by $f_{j}(x, g)=D\left(d_{j} \alpha(x)\right) \lambda^{2 j+1} \rho(g)$ $(x \in S((s+2) L), g \in G)$. Then $f_{j}(-x,-g)=f_{j}(x, g)$ and so $f_{j}$ may be viewed as a map from $S((s+2) L) \times{ }_{\Gamma} G$ to $U\left(2^{s+1}\right)$. We write $\tilde{\beta}\left(\lambda^{2 j+1} \rho\right)$ for $\beta\left(f_{j}\right) \in$ $\tilde{K}_{\Gamma}^{-1}(S((s+2) L) \times G)$. Then we have

\section{Lemma 4.4.}

$$
K_{\Gamma}^{*}(S((s+2) L) \times G)=\boldsymbol{Z}[\tilde{\gamma}] /\left(\tilde{\gamma}^{2}+2 \tilde{\gamma}, 2^{s+1} \tilde{\gamma}\right) \otimes \Lambda\left(\tilde{\nu}, \tilde{\beta}\left(\lambda^{1} \rho\right), \cdots, \tilde{\beta}\left(\lambda^{n} \rho\right)\right) / I
$$

where $I=(\tilde{\gamma} \otimes \tilde{\nu})$.

Proof. Let $i: S((k-1) L) \subset S(k L)$ be the embedding as in $\S 1$ where $1 \leq$ $k \leq s+2$. Denote by the same symbol the restriction of $\tilde{\beta}\left(\lambda^{\ell} \rho\right)$ to $K_{\Gamma}^{*}(S(k L) \times G)$ by $i^{*}$. Then there is a natural morphism $K_{\Gamma}^{*}(X) \otimes E \rightarrow K_{\Gamma}^{*}(X \times G)$ induced by the cross product where $E=\Lambda\left(\tilde{\beta}\left(\lambda^{1} \rho\right), \cdots, \tilde{\beta}\left(\lambda^{n} \rho\right)\right)$. We show that this is an isomorphism when $X=S((s+2) L)$.

Let us view as $S(L)=\{D(n x) ! x \in S(L)\} \subset G$. Then the multiplication on $G$ induces an equivariant homeomorphism $S(L) \times G \approx S(L) \times \phi(G)$ where $\phi(G)$ denotes $G$ with trivial $\Gamma$-action. This yields $K_{\Gamma}^{*}(S(L) \times G) \cong K^{*}\left(S^{1}\right) \otimes K^{*}(G)$ where $S^{1}=S(L) / \Gamma$. And we see that $\tilde{\nu}$ and $\tilde{\beta}\left(\lambda^{\ell} \rho\right)$ correspond to $\mu \otimes 1$ and $1 \otimes \beta\left(\lambda^{\ell} \rho\right) \bmod \boldsymbol{Z} \cdot \mu \otimes 1$ under this isomorphism. Hence $K_{\Gamma}^{*}(S(L) \times G) \cong K_{\Gamma}^{*}$ $(S(L)) \otimes E$.

According to (1.1), $S(k L) \times X / S((k-1) L) \times X \approx \Sigma^{(k-1) L} \wedge(S(L) \times X)_{+}$for any $\Gamma$-space $X$. Hence identifying the Thom isomorphism we have an exact sequence

$$
\cdots \rightarrow K_{\Gamma}^{*}(S(L) \times X) \rightarrow K_{\Gamma}^{*}(S(k L) \times X) \rightarrow K_{\Gamma}^{*}(S((k-1) L) \times X) \rightarrow \cdots
$$


from the exact sequence in $K_{\Gamma}$-theory for the pair $(S(k L) \times X, S((k-1) L) \times X)$. Making use of this sequence when $X=G$ and a single point, and using the above result when $k=1$ it follows by induction on $k$ that the above morphism $K_{\Gamma}^{*}$ $(S(k L)) \otimes E \rightarrow K_{\Gamma}^{*}(S(k L) \times G)$ is an isomorphism for each $k$. This completes the proof.

Proof of Theorem 4.1. Let $\tilde{\beta}(k) \in K_{\Gamma}^{-1}(S((s+2) H) \times G)$ be the element obtained by replacing, for example, $\lambda^{1} \rho$ by $\kappa$ in $\tilde{\beta}\left(\lambda^{1} \rho\right)$. Then by definition we see that $\tilde{\beta}(\kappa)=a_{0} \tilde{\beta}\left(\lambda^{1} \rho\right)+\cdots+a_{n-1} \tilde{\beta}\left(\lambda^{2 n-1} \rho\right)$. Observe the exact sequence of (4.2). Directly from the definition, it then follows that

$$
\begin{aligned}
& I\left(\beta\left(\lambda^{2 i} \rho\right)\right)=1 \otimes \tilde{\beta}\left(\lambda^{2 i} \rho\right), I\left(\beta\left(\kappa_{\jmath}\right)\right)=1 \otimes\left(\tilde{\beta}\left(\lambda^{2 j+1} \rho\right)-d_{\jmath} \tilde{\beta}(\kappa)\right), \\
& I(\beta(\kappa, 2))=(\tilde{\gamma}+2) \otimes \tilde{\beta}(\kappa)+\tilde{\nu} \otimes 1
\end{aligned}
$$

and

$$
\Delta\left(1 \otimes \tilde{\beta}\left(\lambda^{2 \jmath+1} \rho\right)\right)=-d_{j}, \Delta(1 \otimes \tilde{\beta}(\kappa))=-1, \Delta(\tilde{\nu} \otimes 1)=\tilde{\gamma}+2 .
$$

Using these formulas together with (4.3) and Lemma 4.4 we see from the exactness of (4.2) and the argument similar to that of $\bar{\beta}\left(\bar{\chi}_{j}\right)$ 's in $\S 3$ that $\Lambda\left(\beta\left(\chi_{j}\right)(1 \leq\right.$ $j \leq t)$ ) is a subalgebra of $K^{*}(P G)$ with $t=[(n-1) / 2]-1$. And further inspection proves the theorem easily.

\section{§5. Calculation of $K O^{*}(P G)$}

We ready to calculate $K O^{*}(P G)$. We proceed in nearly the same way as for $K^{*}(P G)$. In order to state our theorem we first prepare a few notations.

Since each $\bar{\chi}$ is a linear combination of $\lambda_{C}^{2 k+1} \bar{\rho}$ 's over $\boldsymbol{Z}$ and there holds $\bar{\beta}\left(\lambda_{C}^{2 k+1} \bar{\rho}\right)^{2}=\eta_{1} \beta\left(\lambda_{c}^{4 k+2} \bar{\rho}\right)$ for any $k$, it is seen that there exists a linear combination $\varphi_{j}$ of $\lambda_{c}^{2 k} \bar{\rho}^{\prime}$ s with non-negative integers as coefficients satisfying $\bar{\beta}\left(\bar{\chi}_{j}\right)^{2}=\eta_{1} \beta\left(\varphi_{j}\right)$. Set $\alpha=a_{0}^{2} \lambda_{c}^{2} \bar{\rho}+\cdots+a_{n-1}^{2} \lambda_{c}^{4 n-2} \bar{\rho} \quad$ where $a_{k}$ 's are as in $\S 3$. Then we see analogously that there holds $\bar{\beta}(\bar{\kappa})^{2}=\eta_{1} \beta(\alpha)$. Also we need an additional element of dimension -6 , denoted by $\bar{\zeta} \in K O^{-6}(P G)$, which is given in (5.9) and (6.8) below. Further, let us set

$$
s=4 r+u \quad(0 \leq u \leq 3)
$$

where $s=\nu_{2}(n)$ as before. Then we have the following

Theorem 5.1. With the above notation

$$
\begin{gathered}
K O^{*}(P S p(n))=K O^{*}(+)[\bar{\gamma}, \bar{\zeta}] /\left(\bar{\gamma}^{2}+2 \gamma, 2^{s+2} \bar{\gamma}, \bar{\zeta}^{2}\right) \\
\otimes_{K O^{*}(+)} \Lambda_{K O^{*}(+)}\left(\bar{\beta}(\bar{\kappa}, 2), \beta\left(\lambda_{C}^{2 i} \bar{\rho}\right), \bar{\beta}\left(\bar{\chi}_{j}\right)(1 \leq i \leq[n / 2], 1 \leq j \leq[(n-1) / 2]-1)\right) / I
\end{gathered}
$$


where I is the ideal generated by the elements

$2^{s} \eta_{4} \bar{\gamma}, 2 \bar{\zeta}, \eta_{4} \bar{\zeta}, \eta_{1}^{2} \bar{\zeta}-2^{s+v+1} \bar{\gamma}, \bar{\beta}(\bar{\kappa}, 2)^{2},\left(\beta\left(\lambda_{C}^{2 i} \bar{\rho}\right)^{2}-\eta_{1}\left(\beta\left(\lambda_{C}^{4 \imath} \bar{\rho}\right)+\left(\begin{array}{c}2 n \\ 2 i\end{array}\right) \beta\left(\lambda_{C}^{2 \imath} \bar{\rho}\right)\right)\right)$ 's,

$$
\left(\bar{\beta}\left(\bar{\chi}_{\jmath}\right)^{2}-\eta_{1} \beta\left(\varphi_{j}\right)\right) \text { 's, } \bar{\gamma} \bar{\zeta}-\eta_{1} \bar{\beta}(\bar{\kappa}, 2), \bar{\gamma} \bar{\beta}(\bar{\kappa}, 2), \bar{\zeta} \bar{\beta}(\bar{\kappa}, 2)-\eta_{1}^{2} \bar{\gamma} \beta(\alpha)
$$

with $v=0(u=0,1), v=1$ (otherwise).

The proof of the theorem is divided into the four cases according as $0 \leq u$ $\leq 3$.

We first study $K O_{\Gamma}^{*}(S((8 r+u+4) H) \times G)$. By $(2.5), \widetilde{K O}^{-4}\left(P^{8 r+u+3}\right)=$ $\boldsymbol{Z} / 2^{4 r+u} \cdot \eta_{4} \bar{\gamma}$. So by using (1.2) we have $2^{4 r+u} \bar{\gamma} \otimes \boldsymbol{H}=0$ which implies that there is a quaternionic $\Gamma$-bundle isomorphism $S((8 r+u+4) H) \times\left(2^{4 r+u} H \otimes \boldsymbol{H} \oplus M\right) \cong$ $S((8 r+u+4) H) \times\left(\boldsymbol{H}^{2^{4 r+u}} \oplus M\right)$ for some quaternionic $\Gamma$-module $M$. This gives rise to a base point preserving map $\bar{\alpha}: S((8 r+u+4) H) \rightarrow S p\left(2^{4 r+u}+m\right)$ for some $m \geq 0$ satisfying $\bar{\alpha}(-x)=\bar{\alpha}(x) D\left(-I_{1}, I_{2}\right)(x \in S((8 r+u+4) H))$ where $I_{1} \in$ $S p\left(2^{4 r+u}\right)$ and $I_{2} \in S p(m)$ denote the identities. For simplicity assume that $m=0$. Then since $\operatorname{dim}_{H} \lambda^{2 j+1} \bar{\rho}=2^{4 r+u} d$, we can define a map $\bar{f}_{j}: S((8 r+u+4) H)$ $\times G \rightarrow S p\left(2^{4 r+u}\right)$ by $\bar{f}_{j}(x, g)=D(d, \bar{\alpha}(x)) \lambda^{2 j+1} \bar{\rho}(g)$ for $0 \leq j \leq n-1$. Clearly this map satisfies $\bar{f}_{j}(-x,-g)=\bar{f}_{j}(x, g)$ and so defines naturally an element $\beta\left(\bar{f}_{j}\right) \in$ $\widetilde{K S} p_{\Gamma}^{-1}(S((8 r+u+4) H) \times G)$. We put

$$
\tilde{\tilde{\beta}}\left(\lambda_{C}^{2+1} \bar{\rho}\right)=\sigma \wedge_{C} \beta(\bar{f}) \in \widetilde{K O}_{\Gamma}^{-5}(S((8 r+u+4) H) \times G)(0 \leq j \leq n-1) .
$$

In general, for $a \in K O^{*}\left(P^{k}\right)$ and $b \in K O^{*}(P G)$ we write

$$
\tilde{a}=p_{1}^{*}(a), \tilde{b}=p_{2}^{*}(b) \in K O_{\Gamma}^{*}(S(k H) \times G)
$$

where $p_{1}$ and $p_{2}$ denote the canonical projections from $S(k H) \times G$ to the 1 st and 2 nd factors. Then we have

Lemma 5.2. $K O_{\Gamma}^{*}(S((8 r+u+4) H) \times G) \cong K O^{*}\left(P^{8 r+u+3}\right) \otimes_{K O^{*}(+)} E$ for $0 \leq u \leq 3$ where $E=\Lambda_{K O^{*}(+)}\left(\tilde{\beta}\left(\lambda_{C}^{22} \bar{\rho}\right), \tilde{\bar{\beta}}\left(\lambda_{C}^{2 j+1} \bar{\rho}\right)(1 \leq i \leq[n / 2], 0 \leq j \leq\right.$ $[(n-1) / 2]))$ with the relations $\tilde{\beta}\left(\lambda_{c}^{2 i} \bar{\rho}\right)^{2}=\eta_{1}\left(\tilde{\beta}\left(\lambda_{c}^{42} \bar{\rho}\right)+\left(\begin{array}{c}2 n \\ 2 i\end{array}\right) \tilde{\beta}\left(\lambda_{c}^{2 i} \bar{\rho}\right)\right)$ and $\tilde{\beta}\left(\lambda_{C}^{2 j+1} \bar{\rho}\right)^{2}=\eta_{1} \tilde{\beta}\left(\lambda_{C}^{4 j+2} \bar{\rho}\right)$.

Proof. We give a natural morphism $T_{k}: K O^{*}\left(P^{k-1}\right) \otimes_{K O^{*}(+)} E \rightarrow K O_{\Gamma}^{*}(S(k H)$ $\times G)$ by $T_{k}(x \otimes y)=p_{1}^{*}(x) \hat{\otimes} y\left(x \in K O^{*}\left(P^{k-1}\right), y \in E\right)$ for $1 \leq k \leq 8 r+u+4$ and show that $T_{8 r+u+4}$ is an isomorphism by induction by $k$.

When $1 \leq k \leq 4$ we can regard as $S(k H) \subset \boldsymbol{H}$ canonically and identify any $x \in S(k H)$ with $D(n x) \in G$. Then $S(k H)$ becomes a $\Gamma$-invariant subspace of $G$ 
and so we have $S(k H) \times{ }_{\Gamma} G \approx P^{k-1} \times \phi(G)$ which is induced by, for example, the assignment $(x, g) \mapsto(\pi(x), D(n x) g)(x \in S(k H), g \in G)$. Moreover $K O^{*}(G)$ is free over $K O^{*}(+)$. So we see that this homeomorphism yields an isomorphism $K O_{\Gamma}^{*}(S(k H) \times G) \cong K O^{*}\left(P^{k-1}\right) \otimes_{K O^{*}(+)} K O^{*}(G)$ which sends $\tilde{\beta}\left(\lambda_{c}^{2 i} \bar{\rho}\right)$ and $\bar{\beta}\left(\lambda_{C}^{2 j+1} \bar{\rho}\right)$ to $1 \otimes \beta\left(\lambda_{C}^{2 t} \bar{\rho}\right)$ and $1 \otimes \bar{\beta}\left(\lambda_{C}^{2 j+1} \bar{\rho}\right) \bmod K O^{*}\left(P^{k-1}\right)$ respectively. Hence it is immediate that $T_{k}$ is an isomorphism for $1 \leq k \leq 4$.

Let $h_{\Gamma}^{*}(X)$ denote either $K O_{\Gamma}^{*}(X \times G)$ or $K O_{\Gamma}^{*}(X) \otimes_{K O^{*}(+)} E$. Apply $h_{\Gamma}^{*}$ to the cofibration $S(4(k-1) H) \rightarrow S(4 k H) \rightarrow \Sigma^{4(k-1) H} \wedge S(4 H)_{+}$. Then we get an exact sequence

$$
\cdots \rightarrow h_{\Gamma}^{*}(S(4 H)) \rightarrow h_{\Gamma}^{*}(S(4 k H)) \rightarrow h_{\Gamma}^{*}(S(4(k-1) H)) \rightarrow \cdots
$$

under the identification of the Thom isomorphism $\tilde{h}_{\Gamma}^{*}\left(\Sigma^{4(k-1) H^{+4}} \wedge S(4 H)_{+}\right) \cong h_{\Gamma}^{*}$ $(S(4 H))$. Compare these two exact sequences by using $T_{k}$. Then it is easily seen by induction on $k$ that $T_{8 r+4}$ is an isomorphism because so is $T_{4}$. Next again by applying $h_{\Gamma}^{*}$ to the cofibration $S((8 r+4) H) \rightarrow S((8 r+u+4) H) \rightarrow \Sigma^{(8 r+4) H} \wedge$ $S(u H)$ + we have an exact sequence

$$
\cdots \rightarrow h_{\Gamma}^{*}(S(u H)) \rightarrow h_{\Gamma}^{*}(S((8 r+u+4) H)) \rightarrow h_{\Gamma}^{*}(S((8 r+4) H)) \rightarrow \cdots
$$

and so in a way similar to the above we can check that $T_{8 r+u-4}$ is an isomorphism since so are $T_{u}$ and $T_{8 r+4}$. This completes the proof.

First we consider the cases $u=0,1$. Similarly to the complex case the pair $(B((8 r+u+4) H) \times G, S((8 r+u+4) H) \times G)$ gives rise to an exact sequence

$$
\cdots \rightarrow K O^{*}(P G) \stackrel{I}{\rightarrow} K O_{\Gamma}^{*}(S((8 r+u+4) H) \times G) \stackrel{\Delta}{\rightarrow} \widetilde{K O_{\Gamma}^{*}}\left(\Sigma^{u H} \wedge G_{+}\right) \stackrel{J}{\rightarrow} \cdots
$$

under the identification of the Thom isomorphism $\widetilde{K O_{\Gamma}^{*}}\left(\Sigma^{(8 r+u+4) H+4} \wedge G_{+}\right) \cong \widetilde{K O_{\Gamma}^{*}}$ $\left.\Sigma^{u H} \wedge G_{+}\right)$where there holds

$$
\Delta(x I(y))=\Delta(x) y
$$

Moreover we can define an element $\tilde{\beta}(\bar{\kappa}) \in K O_{\Gamma}^{-5}(S((8 r+u+4) H) \times G)$ similar to $\tilde{\beta}(\kappa)$ which satisfies $\tilde{\bar{\beta}}(\tilde{\kappa})=a_{0} \tilde{\bar{\beta}}\left(\lambda_{c}^{1} \bar{\rho}\right)+\cdots+a_{n-1} \tilde{\bar{\beta}}\left(\lambda_{c}^{2 n-1} \tilde{\rho}\right)$. Then by definition we have the following formulas analogous to (4.5) as for $I$.

$$
\begin{gathered}
I\left(\beta\left(\lambda_{c}^{2 i} \bar{\rho}\right)\right)=\tilde{\beta}\left(\lambda_{c}^{22} \bar{\rho}\right), I\left(\bar{\beta}\left(\bar{\kappa}_{j}\right)\right)=\tilde{\bar{\beta}}\left(\lambda_{c}^{2 j+1} \bar{\rho}\right)-d_{j} \tilde{\bar{\beta}}(\bar{\kappa}), \\
I(\tilde{\beta}(\tilde{\kappa}, 2))=(\tilde{\gamma}+2) \tilde{\bar{\beta}}(\bar{\kappa})+\left\{\begin{array}{l}
\tilde{\nu}(u=0) \\
0(u=1) .
\end{array}\right.
\end{gathered}
$$

Using this we can show that (5.3) becomes as follows. 


\section{Lemma 5.6.}

$$
0 \rightarrow K O^{*}(P G) \stackrel{I}{\rightarrow} K O_{\Gamma}^{*}(S((8 r+u+4) H) \times G) \stackrel{\Delta}{\rightarrow} \widetilde{K O_{\Gamma}^{*}}\left(\Sigma^{u H} \wedge G_{+}\right) \rightarrow 0
$$

for $u=0,1$.

Proof. It suffices to show that $J=0$ in (5.3). Consider the exact sequence of the pair $(B(u H) \times G, S(u H) \times G)$

$$
\cdots \rightarrow \tilde{h}_{\Gamma}^{*}\left(\Sigma^{u H} \wedge G_{+}\right) \stackrel{j_{h}}{\rightarrow} h^{*}(P G) \stackrel{i_{h}}{\rightarrow} h_{\Gamma}^{*}(S(u H) \times G) \stackrel{\delta_{h}}{\rightarrow} \cdots
$$

for $h=K O, K$ where $j_{h}$ and $i_{h}$ denote the homomorphisms induced by obvious inclusions and $\delta_{h}$ the coboundary homomorphism. Then by (1.4)(ii) we have

$$
J(x)=-2^{4 r} \eta_{4} \bar{\gamma} j_{K O}(x)
$$

for $x \in \widetilde{K O_{\Gamma}^{*}}\left(\Sigma^{u H} \wedge G_{+}\right)$. (Note that $\Sigma^{O H}=o_{+}$. .) So the case $u=0$ is immediate from (3.1).

We consider the cases $u=1$. Since $K_{\Gamma}^{*}(S(u H) \times G) \cong K^{*}(G)$, by using Theorem 4.1, (2.1) and (2.4) it can be verified that $\operatorname{Im} j_{K}$ is generated by elements of the form $\gamma \boldsymbol{m}$ where $\boldsymbol{m}$ is a monomial in $\beta\left(\lambda^{2 i} \rho\right)$ 's, $\beta\left(\kappa_{\mathrm{g}}\right)$ 's. Together with this we also observe the Bott sequence (1.3) for $P G$. Consider the order of elements of $K O^{*}(P G)$ using (5.5), (2.5), (3.1) and noting that $c(\bar{\gamma})=\gamma, c\left(\beta\left(\lambda_{c}^{2 l} \bar{\rho}\right)\right)=\beta\left(\lambda^{2 i} \rho\right)$, $c\left(\bar{\beta}\left(\bar{\kappa}_{\jmath}\right)\right)=\mu^{2} \beta\left(\kappa_{\jmath}\right)$. Then we see that there is an element $a \in K O^{-6}(P G)$ such that $c(a)=2^{4 r+1} \mu^{3} \gamma$. And then we see that $\operatorname{Im} j_{K O}$ is generated by elements of the form $\bar{\gamma} \overline{\boldsymbol{m}}, \eta_{4} \bar{\gamma} \overline{\boldsymbol{m}}$, a $\overline{\boldsymbol{m}}$ modulo $\eta_{1} K O^{*}(P G)$ where $\boldsymbol{m}$ is a monomial in $\beta\left(\lambda_{c}^{2 i} \bar{\rho}\right)$ 's, $\bar{\beta}\left(\bar{\kappa}_{\mathrm{g}}\right)$ 's. Now, by definition $\bar{\gamma} a$ is divisible by $\eta_{1}$. So $J=0$ follows from the above equality, since $2^{4 r+1} \eta_{4} \bar{\gamma}=0$ and $\eta_{1} \eta_{4}=0$.

Before proving Theorem 5.1 we make a remark about the subalgebra $E$ of $K O_{\Gamma}^{*}(S((8 r+u+4) H) \times G)$ of Lemma 5.2. Since there hold $\tilde{\beta}\left(\lambda_{C}^{2 j+1} \bar{\rho}\right)=\tilde{\tilde{\beta}}\left(\bar{\kappa}_{j}\right)+$ $d_{j} \tilde{\bar{\beta}}(\bar{\kappa})$ by definition, it is seen that $E$ is generated by $\tilde{\beta}\left(\lambda_{c}^{2 t} \bar{\rho}\right)$ 's, $\tilde{\bar{\beta}}\left(\bar{\kappa}_{j}\right)$ 's and $\tilde{\bar{\beta}}(\bar{\kappa})$. To be exact,

$$
E=\Lambda_{K O^{*}(+)}\left(\tilde{\beta}\left(\lambda_{c}^{2 l} \bar{\rho}\right), \tilde{\beta}\left(\bar{\chi}_{\jmath}\right), \tilde{\bar{\beta}}(\bar{\kappa}) \quad(1 \leq i \leq[n / 2], 1 \leq j \leq[(n-1) / 2]-1)\right) .
$$

Proof of Cases $u=0,1$. Let us write $R$ for the algebra of the right-hand side of the equality in Theorem 5.1.

By considering (5.7) with a point instead of $G$ we get an element $\omega \in$ $\widetilde{K O}_{\Gamma}\left(\Sigma^{u H}\right)$ satisfying $j_{K O}^{*}(\omega)=(1-H)^{u}$ and $\widetilde{K O}{ }_{\Gamma}^{*}\left(\Sigma^{u H}\right)=K O^{*}(+) \cdot \omega$. Then it follows directly from the definition that

$$
\Delta(\tilde{\tilde{\beta}}(\bar{\kappa}))=-\omega \wedge 1, \Delta(\tilde{\zeta})=\eta_{1} \omega \wedge 1
$$


where $\zeta$ is as in (2.5). Using this and the exactness of Lemma 5.6, the element $\bar{\zeta} \in K O^{-6}(P G)$ of $R$ is uniquely given by

$$
I(\bar{\zeta})=\eta_{1} \tilde{\tilde{\beta}}(\bar{\kappa})+\tilde{\zeta} .
$$

And so by using (5.5), (5.9) and Lemma 5.2 together with (2.5), (2.2) and further by the injectivity of $I$ we can verify that $R$ is a subalgebra of $K O^{*}(P G)$.

It remains to check the fullness of $R$ into $K O^{*}(P G)$. In the case $u=0$ the situation is quite similar to the case of $K^{*}(P G)$ and we have $\operatorname{Im} \Delta=R$, so that $R=K O^{*}(P G)$ by the surjectivity of $\Delta$, by the same discussion using (5.8), (5.9), (5.5) together with (5.4). In the case $u=1$ we have a difference from the case $u=0$ in the 3rd group of the exact sequence of Lemma 5.6. But using (5.8) an analogous argument shows that $\widetilde{K O} \Gamma_{\Gamma}^{*}\left(\Sigma^{H} \wedge G_{+}\right)$is generated by $\omega \wedge 1$ as an $R$ module, so that $\operatorname{Im} j_{K O} \subset R$. To prove the fullness of $R$ into $K O^{*}(P G)$ it therefore suffices to show that $i_{K O}(R)=\operatorname{Im} i_{K O}$ in (5.7). This follows from the equalities

$$
\begin{aligned}
& i_{K O}\left(\beta\left(\lambda_{c}^{2 l} \bar{\rho}\right)\right)=\tilde{\beta}\left(\lambda_{C}^{2 i} \bar{\rho}\right), i_{K O}\left(\bar{\beta}\left(\bar{\kappa}_{j}\right)\right)=\tilde{\bar{\beta}}\left(\lambda_{C}^{2 j+1} \bar{\rho}\right)-d_{j} \tilde{\beta}(\bar{\kappa}), \\
& i_{K O}(\bar{\beta}(\bar{\kappa}, 2))=2 \tilde{\beta}(\bar{\kappa}), i_{K O}(\bar{\zeta})=\eta_{1} \tilde{\bar{\beta}}(\bar{\kappa}), \delta_{K O}(\tilde{\beta}(\bar{\kappa}))=2^{4 r+1} \eta_{4} \omega \wedge 1
\end{aligned}
$$

together with $\delta_{K O}\left(x i_{K O}(y)\right)=\delta_{K O}(x) y$ and an inspection of the complexification form of these formulas using the results of $\S 4$.

\section{§ 6. Proof of Cases $u=2,3$}

We begin by calculating $K O_{\Gamma}^{*}(S((8 r+10) H) \times G)$. Through the homeomorphism of (1.1) we have a cofibration $S((8 r+4) H) \times G \rightarrow S((8 r+10) H) \times G \rightarrow$ $\Sigma^{(8 r+4) H} \wedge(S(6 H) \times G)_{+}$. Under the identification of the Thom isomorphism for $\Sigma^{(8 r+4) H+4}$ and the isomorphism of Lemma 5.2 this gives the following exact sequence:

$$
\cdots \rightarrow K O^{*}\left(P^{5}\right) \otimes_{K O^{*}(+)} E \stackrel{J}{\rightarrow} K O_{\Gamma}^{*}(S((8 r+10) H) \times G) \stackrel{I}{\rightarrow} K O^{*}\left(P^{8 r+3}\right) \otimes_{K O^{*}(+)} E \stackrel{\delta}{\rightarrow} \cdots
$$

Using this sequence we can get the above group. We set

$$
w=4-u
$$

and consider generators. As before let us write $\tilde{x}$ for the element induced from a generator $x$ of each factor of $S((8 r+10) H) \times G$. Let $w \kappa_{R}$ and $2 w \bar{\kappa}$ denote the direct sums of $w$ and $2 w$ copies of the realification of $\kappa_{R}$ and the $\bar{\kappa}$ respectively. Then these maps give rise to elements 


$$
\beta\left(w \kappa_{R}\right) \in \widetilde{K O}_{\Gamma}^{-1}(S((8 r+10) H) \times G) \text { and } \bar{\beta}(2 w \bar{\kappa}) \in \widetilde{K O}_{\Gamma}^{-5}(S((8 r+10) H) \times G)
$$

similarly to, for example, $\tilde{\tilde{\beta}}\left(\lambda_{C}^{2 \jmath+1} \bar{\rho}\right)$ in $\S 5$ by making use of the maps arising from the equalities $2^{4 r+5} \bar{\gamma}=0 \in \widetilde{K O}\left(P^{8 r+9}\right)$ and $2^{4 r+4} \eta_{4} \bar{\gamma}=0 \in \widetilde{K O}{ }^{-4}\left(P^{8 r+9}\right)$ respectively.

In the construction of $\tilde{\beta}(\bar{\kappa}) \in K O_{\Gamma}^{-5}(S((8 r+4) H) \times G)$, we may take the $\Gamma$ $\operatorname{map} S((8 r+4) H) \subset S((8 r+6) H) \rightarrow S p\left(2^{4 r+3}\right)$ arising from the equality $2^{4 r+2} \eta_{4} \bar{\gamma}$ $=0 \in \widetilde{K O^{-4}}\left(P^{8 r+5}\right)$ as the $\Gamma$-map corresponding to $\bar{\alpha}$ mentioned after Theorem 5.1 because of $\widetilde{K O}-5\left(P^{8 r+5}\right)=0$. Then we see

$$
\delta(1 \otimes \tilde{\beta}(\bar{\kappa}))=-2^{u-1} \bar{\gamma} \otimes 1,
$$

so that there is an element $\tilde{\zeta} \in K O_{\Gamma}^{-6}(S((8 r+10) H) \times G)$ such that

$$
I(\tilde{\zeta})=1 \otimes \eta_{1} \tilde{\tilde{\beta}}(\bar{\kappa})
$$

From a direct construction of $\tilde{\zeta}$ we see that it may satisfy

$$
2 \tilde{\zeta}=\eta_{1}^{2} \tilde{\zeta}=\eta_{4} \tilde{\zeta}=0 \text { and } \phi(\tilde{\zeta})=1 \times \eta_{1} \bar{\beta}(\phi(\bar{\kappa}))
$$

where $\phi$ denotes the same forgetful functor as in $\S \S 1$ and 4 . Furthermore we put

$$
\epsilon=J(\bar{\nu} \otimes \tilde{\bar{\beta}}(\bar{\kappa})) \text { and } \epsilon^{\prime}=J\left(\bar{\nu}^{\prime} \otimes \tilde{\tilde{\beta}}(\bar{\kappa})\right) \text {. }
$$

Then we have

Lemma 6.4. With the above notation $K O_{\Gamma}^{*}(S((8 r+10) H) \times G)$ is an algebra over $K O^{*}(+)$ generated by

$1, \tilde{\gamma}, \tilde{\beta}\left(\lambda_{c}^{2 i} \tilde{\rho}\right)(1 \leq i \leq[n / 2]), \tilde{\bar{\beta}}\left(\chi_{\jmath}\right)(1 \leq j \leq[(n-1) / 2]-1)$,

$\tilde{\beta}(\bar{\kappa}, 2), \tilde{\nu}, \tilde{\nu}^{\prime}, \epsilon, \epsilon^{\prime}, \beta\left(2 w \kappa_{R}\right), \bar{\beta}(2 w \bar{\kappa}), \tilde{\zeta}$

with the relations

$$
\begin{aligned}
& 2^{s+2} \bar{\gamma}=2^{s} \eta_{4} \bar{\gamma}=2 \tilde{\zeta}=0, \tilde{\beta}\left(\lambda_{c}^{2 \imath} \bar{\rho}\right)^{2}=\eta_{1}\left(\tilde{\beta}\left(\lambda_{C}^{4 i} \bar{\rho}\right)+\left(\begin{array}{c}
2 n \\
2 i
\end{array}\right) \tilde{\beta}\left(\lambda_{C}^{22} \bar{\rho}\right)\right) ' s, \\
& \tilde{\bar{\beta}}\left(\bar{\chi}_{j}\right)^{2}=\eta_{1} \tilde{\beta}\left(\varphi_{j}\right) \text { 's, } \tilde{\bar{\beta}}(\bar{\kappa}, 2)^{2}=\tilde{\nu}^{2}=\tilde{\nu}^{2}=\epsilon^{2}=\epsilon^{\prime 2}=\beta\left(w \kappa_{R}\right)^{2}=\bar{\beta}(2 w \bar{\kappa})^{2}=\tilde{\zeta}^{2}=0, \\
& \tilde{\gamma} \tilde{\beta}(\bar{\kappa}, 2)=\tilde{\gamma} \tilde{\nu}=\tilde{\gamma} \tilde{\nu}^{\prime}=\tilde{\gamma} \epsilon=\tilde{\gamma} \epsilon^{\prime}=0, \tilde{\nu} \tilde{\nu}^{\prime}=\epsilon \epsilon^{\prime}=0, \tilde{\nu} \bar{\beta}(2 w \bar{\kappa})=2 w \epsilon, \\
& \tilde{\mathcal{\nu}} \tilde{\beta}\left((\bar{\kappa}, 2)=2 \epsilon, \tilde{\nu}^{\prime} \bar{\beta}(2 w \bar{\kappa})=2 w \epsilon^{\prime}, \tilde{\nu}^{\prime} \tilde{\bar{\beta}}(\bar{\kappa}, 2)=2 \epsilon^{\prime}, \epsilon \tilde{\bar{\beta}}(\bar{\kappa}, 2)=\epsilon^{\prime} \tilde{\bar{\beta}}(\bar{\kappa}, 2)=0\right. \text {, } \\
& \epsilon \tilde{\zeta}=\epsilon^{\prime} \tilde{\zeta}=0, \tilde{\zeta} \tilde{\beta}(\bar{\kappa}, 2)=\eta_{1}^{2} \tilde{\gamma} \tilde{\beta}(\alpha), \tilde{\gamma} \tilde{\zeta}=\eta_{1} \tilde{\beta}(\bar{\kappa}, 2), \epsilon \beta\left(w \kappa_{R}\right)=\epsilon^{\prime} \beta\left(w \kappa_{R}\right)=0,
\end{aligned}
$$




$$
\begin{aligned}
& \tilde{\zeta} \beta\left(w \kappa_{R}\right)=0, \beta\left(w \kappa_{R}\right) \bar{\beta}(2 w \bar{\kappa})=\beta\left(w \kappa_{R}\right) \bar{\beta}(\bar{\kappa}, 2)=\bar{\beta}(2 w \bar{\kappa}) \bar{\beta}(\bar{\kappa}, 2)=0, \\
& \epsilon \bar{\beta}(2 w \bar{\kappa})=\epsilon^{\prime} \bar{\beta}(2 w \bar{\kappa})=0, \tilde{\zeta} \bar{\beta}(2 w \bar{\kappa})=0, \tilde{\nu} \beta\left(w \kappa_{R}\right)=\tilde{\nu}^{\prime} \bar{\beta}(2 w \bar{\kappa}), \\
& \tilde{\nu}^{\prime} \beta\left(w \kappa_{R}\right)=\tilde{\nu} \bar{\beta}(2 w \bar{\kappa}), \epsilon \tilde{\bar{\nu}}=\epsilon^{\prime} \tilde{\bar{\nu}}=\epsilon_{\bar{\nu}^{\prime}}=\epsilon^{\prime} \tilde{\nu}^{\prime}=0, \eta_{1} \beta\left(w \kappa_{R}\right)=\eta_{1} \tilde{\nu}^{\prime}=0, \\
& \eta_{1} \tilde{\bar{\nu}}=2^{4 r+4} \tilde{\bar{\gamma}}, \eta_{1} \epsilon=\tilde{\nu} \tilde{\zeta}=2^{s-1} \eta_{4} \tilde{\gamma} \beta\left(w \kappa_{R}\right), \eta_{1} \epsilon^{\prime}=\tilde{\nu}^{\prime} \tilde{\zeta}=2^{s} \tilde{\gamma} \beta\left(w \kappa_{R}\right), \eta_{1} \bar{\beta}(2 w \bar{\kappa})=0, \\
& \eta_{1}^{2} \tilde{\zeta}=\eta_{4} \tilde{\zeta}=0, \eta_{4} \tilde{\nu}=2 \tilde{\nu}^{\prime}, \eta_{4} \tilde{\nu}^{\prime}=2 \tilde{\bar{\nu}}, \eta_{4} \epsilon=2 \epsilon^{\prime}, \eta_{4} \epsilon^{\prime}=2 \epsilon, \eta_{4} \bar{\beta}(2 w \bar{\kappa})=2 \beta\left(w \kappa_{R}\right), \\
& \eta_{4} \beta\left(w \kappa_{R}\right)=2 \bar{\beta}(2 w \bar{\kappa}), \eta_{4} w \tilde{\beta}(\tilde{\kappa}, 2)=(\tilde{\bar{\gamma}}+2) \beta\left(w \kappa_{R}\right), 2 w \tilde{\beta}(\bar{\kappa}, 2)=(\tilde{\gamma}+2) \bar{\beta}(2 w \bar{\kappa}) .
\end{aligned}
$$

Proof. By construction we have

$$
\begin{aligned}
& J(1)=-2^{4 r} \eta_{4} \tilde{\bar{\gamma}}, J(\bar{\nu} \otimes 1)=\tilde{\nu}, J\left(\bar{\nu}^{\prime} \otimes 1\right)=\tilde{\nu}^{\prime}, \delta(\bar{\nu} \otimes 1)=(\bar{\gamma}+2) \otimes 1, \\
& \delta(\zeta \otimes 1)=\eta_{1} \otimes 1, I(\tilde{\tilde{\gamma}})=\bar{\gamma} \otimes 1, I(\tilde{\bar{\beta}}(\bar{\kappa}, 2))=(\tilde{\gamma}+2) \otimes \tilde{\beta}(\bar{\kappa}), \\
& I(\bar{\beta}(2 w \bar{\kappa}))=2 w \otimes \tilde{\beta}(\bar{\kappa}), I\left(\beta\left(w \kappa_{R}\right)\right)=\eta_{4} w \otimes \tilde{\bar{\beta}}(\bar{\kappa}) .
\end{aligned}
$$

Further, by noting (1.2) and the equalities $\bar{\gamma} \bar{\nu}=2 \zeta=0$, we get

$$
\begin{aligned}
& J(1 \otimes \tilde{\bar{\beta}}(\bar{\kappa}))=-2^{s-3} \tilde{\gamma} \beta\left(w \kappa_{R}\right), \delta(\bar{\nu} \otimes \tilde{\tilde{\beta}}(\bar{\kappa}))=(\bar{\gamma}+2) \otimes \tilde{\tilde{\beta}}(\bar{\kappa}), \\
& \delta(\zeta \otimes \tilde{\beta}(\bar{\kappa}))=\eta_{1} \otimes \tilde{\beta}(\bar{\kappa}) .
\end{aligned}
$$

Moreover for a monomial $\boldsymbol{m}$ in $\tilde{\beta}\left(\lambda_{c}^{2 i} \bar{\rho}\right)$ 's, $\tilde{\bar{\beta}}\left(\bar{\chi}_{j}\right)$ 's, $\tilde{\bar{\beta}}(\bar{\kappa}, 2)$ we have relations $J(x(1 \otimes \boldsymbol{m}))=J(x) \boldsymbol{m}, I(x \boldsymbol{m})=I(x)(1 \otimes \boldsymbol{m}), \delta(x(1 \otimes \boldsymbol{m}))=\delta(x)(1 \otimes \boldsymbol{m})$ and further there hold $\delta(x I(y))=\delta(x) y$. From these facts together with (6.1) $-(6.3)$, (6.5), (6.6) and the remark stated previous to the proof of the cases $u=0,1$ we can infer that $K O_{\Gamma}^{*}(S((8 r+10) H) \times G)$ is generated by the elements given above.

We next check the algebra structure. Let $X=S(k H), G$ or $S(k H) \times G$ and let $\operatorname{tr}: K O^{*}(\phi(X)) \rightarrow K O_{\Gamma}^{*}(X)$ denote the transfer map. Then it follows that $\operatorname{tr}(\phi(x) y)=x \operatorname{tr}(y)$ and $\phi \operatorname{tr}=2$. By definition $\tilde{\bar{\beta}}(\bar{\kappa}, 2)=\operatorname{tr}(\tilde{\tilde{\beta}}(\phi(\bar{\kappa}))$, so that

$$
\tilde{\tilde{\beta}}(\bar{\kappa}, 2)^{2}=\operatorname{tr}(\phi(\tilde{\tilde{\beta}}(\bar{\kappa}, 2)) \tilde{\tilde{\beta}}(\phi(\bar{\kappa})))=\operatorname{tr}\left(2 \tilde{\tilde{\beta}}(\phi(\bar{\kappa}))^{2}\right)=0
$$

since $\tilde{\bar{\beta}}(\phi(\bar{\kappa}))^{2}$ is divisible by $\eta_{1}$. Similarly $\tilde{\gamma} \tilde{\bar{\beta}}(\bar{\kappa}, 2)=0$ follows because of $\operatorname{tr}(1)=\tilde{\tilde{\gamma}}+2$. Using also the above formulas

$$
\tilde{\zeta} \tilde{\bar{\beta}}(\bar{\kappa}, 2)=\operatorname{tr}(\phi(\tilde{\zeta}) \tilde{\bar{\beta}}(\phi(\bar{\kappa})))=\operatorname{tr}\left(\eta_{1}^{2} \phi(\tilde{\beta}(\alpha))\right)=\eta_{1}^{2} \tilde{\bar{\gamma}} \tilde{\beta}(\alpha)
$$

since $\tilde{\bar{\beta}}(\phi(\bar{\kappa}))^{2}=\eta_{1} \tilde{\beta}(\phi(\alpha))$, and analogously

$$
\eta_{1} \tilde{\bar{\beta}}(\bar{\kappa}, 2)=\operatorname{tr}\left(\eta_{1} \tilde{\bar{\beta}}(\phi(\bar{\kappa}))\right)=\operatorname{tr}(\phi(\tilde{\zeta}))=\tilde{\gamma} \tilde{\zeta} .
$$


By (1.3), $\eta_{1} r(x)=0$ which implies $\eta_{1} \beta\left(w \kappa_{R}\right)=0$. By construction we see that $\eta_{1} \bar{\beta}(2 w \bar{\kappa})=2 \tilde{\zeta}(=0)$. Because $\beta\left(w \kappa_{R}\right)$ is a linear combination of $\beta(\boldsymbol{H}$ $\left.\otimes_{C} \lambda_{C}^{2 j+1} \bar{\rho}\right)$ 's whose squares are zero by $(2.3), \beta\left(w \kappa_{R}\right)^{2}=0$ is immediate.

The other relations are either obtained easily from the construction of the generators or inherited from the relations of the groups on the both sides of the above exact sequence. So we omit the proof of them.

Combining the exact sequence of the pair $(B((8 r+10) H) \times G, S((8 r+10) H)$ $\times G)$ with the Thom isomorphism $\widetilde{K O_{\Gamma}^{*}}\left(\Sigma^{(8 r+10) H} \wedge G_{+}\right) \cong \widetilde{K O_{\Gamma}^{*}}\left(\Sigma^{2 H} \wedge G_{+}\right)$we have an exact sequence

$$
\cdots \rightarrow K O^{*}(P G) \stackrel{I}{\rightarrow} K O_{\Gamma}^{*}(S((8 r+10) H) \times G) \stackrel{\Delta}{\rightarrow} \widetilde{K O_{\Gamma}^{*}}\left(\Sigma^{2 H} \wedge G_{+}\right) \stackrel{J}{\rightarrow} \cdots .
$$

This becomes the following short exact sequence.

\section{Lemma 6.7.}

$$
0 \rightarrow K O^{*}(P G) \stackrel{I}{\rightarrow} K O_{\Gamma}^{*}(S((8 r+10) H) \times G) \stackrel{\Delta}{\rightarrow} \widetilde{K O_{\Gamma}^{*}}\left(\Sigma^{2 H} \wedge G_{+}\right) \rightarrow 0 .
$$

Proof. Consider (5.7) which is of course valid when $u=2,3$. We have an element $a \in K O^{-6}(P G)$ such that $c(a)=2^{s} \mu^{3} \gamma$ and $\bar{\gamma} a$ is divisible by $\eta_{1}$ by using (1.3) and noting that the orders of $\gamma$ and $\eta_{4} \bar{\gamma}$ are $2^{s+1}$ and $2^{s}$ respectively. Further note that the order of $\bar{\gamma}$ is $2^{s+2}$ by (3.1) and Lemma 6.9 and compare $\operatorname{Im} j_{K O}$ with $\operatorname{Im} j_{K}$. Then just as in the case $u=1$ of Lemma 5.6 it is seen that $\operatorname{Im} j_{K O}$ is generated by elements of the form $2 \overline{\boldsymbol{\gamma}} \overline{\boldsymbol{m}}, \eta_{4} \overline{\boldsymbol{m}}$, a $\overline{\boldsymbol{m}}$ modulo $\eta_{1} K O^{*}(P G)$ where $\overline{\boldsymbol{m}}$ is a monomial in $\beta\left(\lambda_{C}^{22} \bar{\rho}\right)$ 's, $\bar{\beta}\left(\bar{\kappa}_{\jmath}\right)$ 's. Because of $J(x)=2^{4 r+3} \bar{\gamma} j_{K O}(x)$, this implies $J=0$ as asserted.

Proof of Cases $u=2$, 3. In order to study the $\Delta$ above we observe (5.7) with $u=2$ in more detail. A short computation gives $\widetilde{K O}_{\Gamma}^{-p}\left(\Sigma^{2 H}\right)$ as follows.

$$
\widetilde{K O}_{\Gamma}^{-2 p}\left(\Sigma^{2 H}\right)=\boldsymbol{Z} \cdot \omega_{p}, \widetilde{K O}_{\Gamma}^{-2 p-1}\left(\Sigma^{2 H}\right)=0
$$

with the relations

$$
\begin{gathered}
(H+1) \omega_{0}=(H+1) \omega_{2}=(H-1) \omega_{1}=(H-1) \omega_{3}=0, \eta_{4} \omega_{0}=2 \omega_{2}, \eta_{4} \omega_{1}=2 \omega_{2} \\
\omega_{0}^{2}=\omega_{2}^{2}=4 \omega_{0}, \omega_{1}^{2}=\omega_{3}^{2}=0, \omega_{0} \omega_{1}=\omega_{0} \omega_{3}=\omega_{1} \omega_{2}=\omega_{1} \omega_{3}=\omega_{2} \omega_{3}=0, \omega_{0} \omega_{2}=4 \omega_{2} .
\end{gathered}
$$

Also these generators satisfy

$$
j_{K O}\left(\omega_{0}\right)=2(1-H), j_{K O}\left(\omega_{1}\right)=\eta_{1}^{2}(1-H), j_{K O}\left(\omega_{2}\right)=\eta_{4}(1-H), j_{K O}\left(\omega_{3}\right)=0 .
$$

Using such notation it follows directly from the construction 


$$
\begin{aligned}
& \Delta(\tilde{\mathcal{\nu}})=\omega_{1} \wedge 1, \Delta\left(\tilde{\tilde{\nu}}^{\prime}\right)=\omega_{3} \wedge 1, \Delta\left(\beta\left(w \kappa_{R}\right)\right)=\omega_{0} \wedge 1, \Delta(\bar{\beta}(2 w \bar{\kappa}))=\omega_{2} \wedge 1, \\
& \Delta(\tilde{\zeta})=0 .
\end{aligned}
$$

The last formula together with Lemma 6.7 shows that the element $\bar{\zeta} \in K O^{-6}(P G)$ of Theorem 5.1 is uniquely defined by

$$
I(\bar{\zeta})=\tilde{\zeta}
$$

And further we have

$$
\Delta\left(\eta_{1} \epsilon\right)=2^{s+1} \omega_{2} \wedge 1, \Delta\left(\eta_{1} \epsilon^{\prime}\right)=2^{s^{\perp}} \omega_{0} \wedge 1
$$

View $i_{K O}$ as the composite

$$
\begin{aligned}
K O^{*}(P G) \stackrel{I}{\rightarrow} K O_{\Gamma}^{*}(S((8 r+10) H) \times G) \stackrel{(1 \times i)^{*}}{\longrightarrow} K O_{\Gamma}^{*}(S(2 H) \times G) \\
\left(\cong K O^{*}\left(S^{1}\right) \otimes_{K O^{*}(+)} E\right)
\end{aligned}
$$

where $i$ is an obvious inclusion of $S(2 H)$ into $S((8 r+10) H)$. Then we see by Lemma 6.4 that these do not belong to $\operatorname{Im} i_{K O}$, namely, the orders of both elements $\eta_{1} \epsilon$ and $\eta_{1} \epsilon^{\prime}$ are 2. Moreover we can verify that

$$
j_{K O} \Delta(\epsilon)=\eta_{1}^{2} \bar{\beta}(\bar{\kappa}, 2), j_{K O} \Delta\left(\epsilon^{\prime}\right)=0, j_{K O}(\tilde{\nu} \tilde{\bar{\beta}}(2 w \bar{\kappa}))=j_{K O}\left(\tilde{\nu}^{\prime} \tilde{\bar{\beta}}(2 w \bar{\kappa})\right)=0 .
$$

Let $R$ be as in $\S 5$. Then it is immediate by Lemma 6.4 and the injectivity of $I$ that $R \subset K O^{*}(P G)$. And from the data above together with $\Delta(x I(y))=\Delta(x) y$ we infer that $\operatorname{Im} i_{K O} \subset R$ since $\Delta$ is surjective. Similarly to the case $u=1$ we also have $\operatorname{Im} i_{K O}=i_{K O}(R)$. Hence we conclude that $K O^{*}(P G)=R$, which completes the proof of Theorem 5.1 .

\section{References}

[1] Atiyah, M. F., K-theory and reality, Quart. J. Math., Oxford, 17 (1966), 367-386.

[2] - K-Theory, Benjamin Inc., 1967.

[3] - Bott periodicity and the index of elliptic operators, Quart. J. Math., Oxford, 74 (1968), 113-140.

[4] Crabb, M. C., $\boldsymbol{Z}_{2}$-Homotopy Theory, London Math. Soc. Lecture Note Series, 44, 1980.

[5] Fujii, M., $K_{0}$-groups of projective spaces, Osaka J. Math., 4 (1967), 141-149.

[6] Held, R. P. and Suter, U., On the unitary $K$-theory of compact Lie groups with finite fundamental group, Quart. J. Math., Oxford, 24 (1973), 343-356.

[7] Hodgkin, L., On the $K$-theory of Lie groups, Topology, 6 (1967), 1-36.

[8] - The equivariant Künneth theorem in $K$-theory, Springer Lecture Notes in Math., 496 (1975), 1-101.

[9] Landweber, P. S., On equivariant maps between spheres with involutions, Ann. of 
Math., 89 (1969), 125-137.

[10] Minami. H., On the $K$-theory of $S O(n)$, Osaka J. Math., 21 (1984), 789-808.

[11] - The real $K$-groups of $S O(n)$ for $n \equiv 3,4$ and $5 \bmod 8$, Osaka J. Math., 25 (1988), $185-211$.

[12] - The real $K$-groups of $S O(n)$ for $n \equiv 2 \bmod 4$, Osaka J. Math., 26 (1989), 299-318.

[13] — On the $K$-theory of $P E_{7}$, Osaka J. Math., 30 (1993), 235-266.

[14] - On the $K$-theory of $P E_{6}$, Osaka J. Math., to appear.

[15] - KO-group of $P S p\left(2^{4 n}\right)$, Math. J. of Okayama Univ., to appear.

[16] Segal, G., Equivariant K-theory, Publ. Math. IHES, 34 (1968), 129-151.

[17] Seymour, R. M., The Real $K$-theory of Lie groups and homogeneous spaces, Quart. J. Math., Oxford 24 (1973), 7-30.

[18] Petrie, T., The $K$-theory of the projective unitary groups, Topology, 6 (1967), 103-115.

[19] Toda, H., Order of the identity class of a suspension space, Ann. of Math., 78 (1963), 300-325. 
